\title{
Resveratrol Administration Increases Transthyretin Protein Levels, Ameliorating AD Features: The Importance of Transthyretin Tetrameric Stability
}

\author{
Luís Miguel Santos, ${ }_{1,2}^{1,}$ Daniela Rodrigues, ${ }^{1,2,3}$ Mobina Alemi, ${ }^{1,2,4}$ Sara Costa Silva, ${ }^{1,2,3}$ Carlos Alexandre Ribeiro, ${ }^{1,2}$ \\ and Isabel Cardoso $0^{1,2 *}$ \\ ${ }^{1}$ Instituto de Biologia Celular e Molecular; ${ }^{2}$ Instituto de Investigação e Inovação em Saúde, Universidade do Porto, Portugal; \\ ${ }^{3}$ Department of Health Sciences, School of Allied Health Technologies, Polytechnic Institute of Porto, Portugal; and ${ }^{4}$ Faculdade de \\ Medicina, Universidade do Porto, Portugal
}

\begin{abstract}
Previous in vivo work showed that resveratrol has beneficial effects in Alzheimer's disease (AD) pathology, resulting in increased expression of transthyretin (TTR). TTR binds amyloid-beta (A $\beta$ ) peptide, avoiding its aggregation and toxicity, and is reduced in the cerebrospinal fluid (CSF) and plasma in AD. Further, resveratrol binds TTR, stabilizing the native TTR tetrameric structure. To further explore the mechanism of neuroprotection conferred by $T R$ in $A D$, resveratrol was administered in the diet to 5- to 8-month-old $A D$ transgenic female mice carrying just 1 copy of the mouse TR gene for 2 months. Effects in brain $A \beta$ burden were evaluated by immunohistochemistry, and total brain A $\beta$ levels by ELISA, showing a striking decrease in both parameters in treated animals. In addition, total brain lipoprotein-related receptor protein 1 (LRP1) levels were increased in treated animals, although its gene expression was unaltered. To further understand the mechanism(s) underlying such improvement in AD features, we measured TTR plasma levels, showing that TTR increased in resveratrol-treated mice, whereas liver TR gene transcription was not altered. These results strengthen the stability hypothesis, which postulates that TTR is unstable in AD, leading to accelerated clearance and lower levels. Therefore, resveratrol, which stabilizes the TTR tetramer results in TTR-normalized clearance, increases the protein plasma levels. In turn, stabilized TTR binds more strongly to A $\beta$ peptide, avoiding its aggregation. Our results represent a step forward in the understanding of the mechanism underlying TTR protection in AD and highlight the possibility of using TTR stabilization as a therapeutic target in AD.
\end{abstract}

Online address: http://www.molmed.org

doi: $10.2119 /$ molmed.2016.00124

\section{INTRODUCTION}

Alzheimer's disease (AD), first described by Alois Alzheimer in 1906 (1), is the most common cause of dementia. With more than 26 million AD cases registered in 2006 (2), the number of patients with AD increases every day, and it is expected that, by 2050, 1 new case of AD will appear every $33 \mathrm{~s}$ (3). AD is characterized by progressive loss of cognitive functions, ultimately leading to death, accompanied by the 2 main pathological features: amyloid plaques and neurofibrillary tangles (NFTs). The first consists of extracellular aggregates of amyloid-beta $(\mathrm{A} \beta)$ peptide, while the latter are intracellular aggregates of abnormally hyperphosphorylated tau protein (4). The $\mathrm{A} \beta$ peptide is generated upon sequential cleavage of the amyloid precursor protein (APP), by $\beta$ - and $\gamma$-secretases, and it is believed that an imbalance between
Address correspondence to Isabel Cardoso, Institute de Biologia Molecular e Celulart Rua Alfredo Allen 208, Porto 4200-135, Portugal. Phone: 00351-220408800; E-mail: icardoso@ibmc.up.pt. Submitted May 9, 2016; Accepted for Publication June 29, 2016; Published Online (www.molmed.org) June 30, 2016.

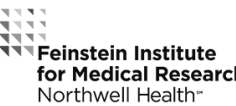

$\mathrm{A} \beta$ production and clearance results in its accumulation in the brain. It was shown that the major pathway for $\mathrm{A} \beta$ clearance is its receptor-mediated transport through the blood-brain barrier (5), which is found to be compromised in $\mathrm{AD}$, with special importance in the sporadic late-onset form (6). In such cases, an increased expression of the receptor for advanced glycation end products (RAGE), the main influx transporter of $\mathrm{A} \beta$ is often observed and/or a decreased expression of the efflux transporters, such as the permeability glycoprotein (P-gp) and the low-density lipoprotein receptor-related protein 1 (LRP1)(7).

Transthyretin (TTR), a 55kDa homotetrameric protein produced mainly in the liver and the choroid plexus, with a well-established role in the transport of thyroxine $\left(\mathrm{T}_{4}\right)$ and retinol, was described 
by Schwarzman and colleagues in the mid-1990s to be the major $A \beta$ binding protein in cerebrospinal fluid (CSF). The authors described that TTR was able to bind and sequester $A \beta$, inhibiting its toxicity and deposition, and that when the sequestration failed, $\mathrm{A} \beta$ would aggregate and form amyloid plaques $(8,9)$, thus suggesting for the first time a neuroprotective role for TTR in AD. More recently, our group has shown that in AD and in mild cognitive impairment (MCI) patients, TTR protein levels are decreased (10) compared with age-matched controls, which is in agreement with a previous observation of lower levels of CSF TTR in AD patients (11). Moreover, TTR levels in the CSF were found to be negatively correlated with formation of senile plaques (12) and disease progression (13), and positively correlated with $\mathrm{A} \beta_{42}$ CSF levels in AD patients (14). Thus it is hypothesized that before $A \beta$ deposition, diminishment of TTR is responsible for increased levels of $A \beta_{42}$, consequently promoting its deposition, and finally, with disease establishment, CSF A $\beta_{42}$ levels decrease due to sequestration in senile plaques. The idea of the neuroprotective role of TTR was strengthened with several in vivo studies using transgenic mice, showing also a negative correlation between TTR and A $\beta$ deposition. When TTR was genetically decreased, mice presented higher levels of $A \beta$ (both $\mathrm{A} \beta_{40}$ and $\mathrm{A} \beta_{42}$ ) in the brain and plasma, accompanied by higher $\mathrm{A} \beta$ deposition $(15,16)$ compared with control mice. This showed that TTR is an important molecule in the clearance of $A \beta$, with recent findings suggesting that TTR promotes $\mathrm{A} \beta$ efflux from the brain to the blood, possibly via LRP1 (17), although the exact mechanism is still unknown.

Given that the familial amyloid polyneuropathy (FAP) is associated with TTR deposition due to loss of stability and function, compounds that stabilize the tetrameric conformation of TTR, such as tafamidis and diflunisal, have been the main drug therapy for FAP patients. As mentioned above, TTR has been indicated as a participant in the development of $\mathrm{AD}$, probably as a consequence of loss of its stability, as in FAP. In fact, we have previously reported that plasma TTR from AD patients presents a decreased ability to carry $\mathrm{T}_{4}$, indicating that this function of TTR is impaired (10). It has also been demonstrated that the more amyloidogenic (and therefore less stable) the variants of TTR are, the less they bind $A \beta$ (18). Thus, we hypothesized that TTR stabilization is a key factor in TTR-A $\beta$ interaction, and subsequently we showed that small compounds that are prone to stabilize TTR increase this interaction in vitro. Importantly, in vivo administration of one such drug resulted in the amelioration of AD features (19). However, results obtained by other authors suggest that it is the monomeric form of TTR that preferentially binds $A \beta$ (20), especially when the peptide is in oligomeric form (21). The monomeric form of TTR was also shown to be the most efficient at inhibiting $A \beta$ fibril formation, although by isotherm titration calorimetry (ITC), the monomeric TTR did not exhibit any sign of binding to A $\beta$, contrary to the tetramer (21). Also, it has been shown that $A \beta$ oligomerrelated toxicity can be neutralized by the binding of TTR (especially monomeric TTR) by promoting their assembly into larger nontoxic species (22). This dual ability to inhibit fibril formation and neutralize oligomer toxicity has been observed in several molecular chaperones, such as $\alpha B$-crystallin, heat shock protein 70, clusterin, haptoglobin and a2-macroglobulin (23), thus TTR has also been noted as a molecule with ATP-independent chaperone activity (22). Although the results point to increased efficiency of the monomeric TTR, most of the data were obtained using an engineered TTR that exists as a stable monomer and whose existence in vivo has not been demonstrated. In fact, Li and colleagues suggest that in vivo, the inhibition of $\mathrm{A} \beta$ fibrillogenesis should be mediated by TTR tetramer binding to $A \beta$ monomer; however, when oligomers are formed, tetrameric and monomeric TTR bind to A $\beta$ oligomers, neutralizing its toxicity (21).
Polyphenolic compounds constitute the main class of secondary metabolites in plants, and are consumed as part of the human diet in significant amounts. Polyphenols can be divided into phenolic acids, lignans, stilbenes and flavonoids, and their contribution to human health has been well described, with multiple biological effects, particularly antioxidant activity. Increased attention has been paid to polyphenols, more specifically the ones present in red wine, after recognition of the "French paradox." This concept refers to the observation that in certain regions of France, lower than expected mortality from coronary heart disease was found (despite high intake of saturated fat), which was correlated to high red wine consumption (24). Moreover, interest in red wine polyphenols as therapeutic agents-in particular resveratrol, due to its strong antioxidant, anti-inflammatory, anti-ischemic and chemopreventive properties-has been growing, with several health benefits described in cancer and cardiovascular prevention, and protection against neurodegenerative disorders such as Alzheimer's, Parkinson's and Huntington's diseases $(25,26)$. More specifically in AD, in vivo administration of resveratrol resulted in reduced neurodegeneration and cognitive decline in mice displaying $\mathrm{AD}$ features $(27,28)$, while in vitro resveratrol lowered the levels of secreted and intracellular $A \beta$ peptides produced from different cell lines by promoting $A \beta$ degradation (29). Interestingly, also using an AD transgenic mice model, it was shown that resveratrol intake increased TTR levels (30). Previous work in our laboratory indicated that resveratrol increases TTR affinity to $A \beta$ (31), probably by stabilizing the TTR native tetramer conformation. It has been described that the binding of resveratrol to TTR is at the $\mathrm{T}_{4}$ binding pocket, although the exact binding site still generates some controversy. Although it was first shown that resveratrol binds TTR in the $\mathrm{T}_{4}$ binding site (32), very recently, Florio and colleagues demonstrated that although resveratrol can compete with $\mathrm{T}_{4}$ (by binding to its site), it possesses a preferential binding site different from that of $\mathrm{T}_{4}(33)$. 
The present work was aimed at investigating the mechanism underlying TTR protection in AD, making use of resveratrol to improve its performance, as an avenue for intervention in this disorder.

\section{MATERIAL AND METHODS}

\section{Chemicals and Proteins}

Resveratrol was purchased from Tokyo Chemical Industry Company and dissolved in DMSO at $200 \mathrm{mM}$, following purity confirmation by high-performance liquid chromatography (HPLC). Synthetic $\mathrm{A} \beta$ (1-42) (Genscript) was dissolved in hexafluoro-2-propanol (HFIP) and kept at room temperature (RT) overnight. The HFIP was removed under a stream of nitrogen and the residue was then dissolved in DMSO at 2mM. Human recombinant wild-type TTR (WT TTR) was produced in a bacterial expression system using Escherichia coli BL21 (34) and purified as previously described (35). Briefly, after growing the bacteria, the protein was isolated and purified by preparative gel electrophoresis after ion exchange chromatography. Protein concentration was determined by the Bradford method (Bio-Rad), using bovine serum albumin (BSA) as standard.

\section{Animals}

The mouse model A $\beta$ PPswe/ PS1A246E/TTR used in this study was generated by crossing A $\beta$ PPswe/ PS1A246E transgenic mice (B6/C3H background) purchased from the Jackson Laboratory with TTR-null mice (TTR-/-) (SV129 background), as previously described (16). In this study, we used cohorts of A $\beta$ PPswe/PS1A246E/TTR+/(hereafter called AD/TTR+/-) female mice 5 to 8 months of age.

For the primary cultures of hepatocytes, nontransgenic TTR-heterozygous (TTR+/-) mice in a SV129 background (36) were used at 2-3 months of age.

Animals were housed in a controlled environment (12-h light/dark cycle; temperature, $22 \pm 2{ }^{\circ} \mathrm{C}$; humidity, 45-65\%), with freely available food and water. All efforts were made to minimize pain and distress. All procedures involving animals were carried out in accordance with national and European Union guidelines for the care and handling of laboratory animals and were performed in compliance with the institutional guidelines and recommendations of the Federation for Laboratory Animal Science Association and approved by the National Authority for Animal Health (Lisbon, Portugal).

\section{Administration of Resveratrol}

$\mathrm{AD} / \mathrm{TTR}+/-$ female mice were divided into 2 groups: the control group, which received a normal diet $(n=7)$, and the treated group, which received a diet incorporating resveratrol $(0.19 \% \mathrm{w} / \mathrm{w})(\mathrm{n}=4)$. The daily consumption of resveratrol was calculated to be $174 \mathrm{mg} / \mathrm{kg} /$ day ( $3.3 \mathrm{~g}$ food per day for a $36 \mathrm{~g}$ mouse). Diets were stored at $4^{\circ} \mathrm{C}$ and animals received fresh food 3 times a week. Resveratrol was administrated for 2 months, and a summary of the animals' ages is presented in Table 1. Control mice were all 5 months of age at the beginning of the experiment.

\section{Primary Hepatocyte Culture}

Primary hepatocytes were derived from nontransgenic TTR+/- mice, as previously described (17). Briefly, a cannula was inserted into the portal vein and perfusion medium (Hank's balanced salt solution [HBSS] $1 \times$ medium containing 0.025 M HEPES and $2 \mathrm{mM}$ ethylenediaminetetraacetic acid [EDTA]) was allowed to perfuse through the liver, with immediate cut of the vena cava. After $10 \mathrm{~min}$, the perfusion medium was substituted with collagenase solution (Williams E medium [WE], Gibco ${ }^{\mathrm{TM}}$ )

Table 1. Summary of the animals treated with resveratrol $(n=4)$.

\begin{tabular}{lcc}
\hline & $\begin{array}{c}\text { Age at beginning } \\
\text { of resveratrol } \\
\text { administration }\end{array}$ & $\begin{array}{c}\text { Age at } \\
\text { euthanization }\end{array}$ \\
\hline Resv1 & 8 months & 10 months \\
Resv2 & 6 months & 8 months \\
Resv3 & 6 months & 8 months \\
Resv4 & 5 months & 7 months \\
\hline
\end{tabular}

containing $10 \%$ fetal bovine serum (FBS), $3 \mathrm{mM} \mathrm{CaCl} 2,0.01 \mathrm{M} \mathrm{HEPES}$ and $1 \mathrm{mg} / \mathrm{mL}$ collagenase type V (Sigma-Aldrich) for another $10 \mathrm{~min}$. The entire perfused liver was then removed to a Petri dish containing isolation medium (WE medium containing 10\% FBS, 2 mM EDTA and $0.01 \mathrm{M}$ HEPES) then proceeded to the next steps of filtering and centrifugation of cell suspension. After counting live cells, $5 \times 10^{5}$ cells were seeded in each well using attachment medium for $3 \mathrm{~h}$, then the medium was changed to stimulation medium. After $24 \mathrm{~h}$ the medium was renewed, and after $48 \mathrm{~h}$, resveratrol $(15 \mu \mathrm{M})$ was added to the media in the respective wells; control cells were kept in stimulation media. Following $24 \mathrm{~h}$ of incubation, supernatants were collected for subsequent TTR protein level analysis.

\section{Tissue Processing}

After resveratrol administration, animals were euthanized following anesthesia with a mixture of ketamine (75 mg/kg) and medetomidine $(1 \mathrm{mg} / \mathrm{kg}$ ) administered by intraperitoneal injection. Brains were removed and bisected longitudinally; each half was either immediately frozen for biochemical analyses or fixed for $24 \mathrm{~h}$ at $4^{\circ} \mathrm{C}$ in $10 \%$ neutral buffered formalin and then transferred to a 30\% sucrose solution for cryoprotection before cryostat sectioning and immunohistochemical analyses. Liver was also collected, immediately frozen and kept at $-80^{\circ} \mathrm{C}$ for mRNA isolation.

\section{A $\beta$ Immunohistochemistry}

$\mathrm{A} \beta$ plaque burden was evaluated by using a monoclonal biotinylated anti-A $\beta_{1-16}$ antibody (6E10) (Covance Research Products, Inc.) to perform free-floating immunohistochemistry of $30 \mu \mathrm{m}$ thick cryostat coronal brain sections. According to a previously described protocol by Oliveira and colleagues (16), free-floating brain sections were washed twice in phosphate buffer saline (PBS) and once in distilled water $\left(\mathrm{dH}_{2} \mathrm{O}\right)$. For partial amyloid denaturation, 70\% formic acid (FA) was used for $15 \mathrm{~min}$ at RT, with gentle agitation. 
After washing in $\mathrm{dH}_{2} \mathrm{O}$ and then PBS, endogenous peroxidase activity was inhibited with $1 \%$ hydrogen peroxide $\left(\mathrm{H}_{2} \mathrm{O}_{2}\right)$ in PBS for $20 \mathrm{~min}$. Following PBS washes, sections were blocked in blocking solution (10\% FBS and $0.5 \%$ Triton $\mathrm{X}-100)$ for $1 \mathrm{~h}$ at RT and then incubated with the biotinylated 6E10 primary antibody (diluted 1:750 in blocking solution) overnight at $4^{\circ} \mathrm{C}$, with gentle agitation. Sections were washed with PBS and incubated in Vectastain ${ }^{\circledR}$ Elite ABC Reagent (Vector Laboratories, Inc.). Sections were washed once more in PBS, followed by development with diaminobenzidine (Sigma-Aldrich, Inc.), mounted on $0.1 \%$ gelatin-coated slides, and left to dry overnight at RT. After dehydration and clearing, slides were coverslipped under Entellan ${ }^{\circledR}$ (Merck and Co., Inc.). A $\beta$ plaque burden was evaluated using Image-Pro Plus software, by analyzing the immunostained area fraction in the hippocampus and cortex (expressed as percentage of analyzed area) of 3 sections per animal, visualized by microscopy (Olympus DP71 microscope).

\section{Brain $A \beta_{42}$ Level Determination}

$\mathrm{A} \beta_{42}$ levels in brain extracts (detergent-soluble and FA-soluble $\mathrm{A} \beta$ ) were evaluated using sandwich ELISA, as previously described. (16) Each halfbrain was homogenized in $1 \mathrm{~mL} 0.1 \%$ Triton X-100 and $2 \mathrm{mM}$ EDTA in $50 \mathrm{mM}$ Tris-buffered saline (TBS) ( $\mathrm{pH} 7.4$ ) with protease inhibitors (GE Healthcare), and centrifuged at $21500 \times \mathrm{g}$ for $15 \mathrm{~min}$ at $4^{\circ} \mathrm{C}$. The supernatant was collected, aliquoted and frozen at $-80^{\circ} \mathrm{C}$ for subsequent analysis (detergent-soluble fraction of brain $A \beta$ ). The FA-soluble fraction of brain $A \beta$ was obtained by homogenizing the pellet with $1 \mathrm{~mL} \mathrm{70 \%} \mathrm{FA} \mathrm{in} \mathrm{dH}_{2} \mathrm{O}$ and centrifugation, as described. The supernatant was collected and neutralized with 1M Tris ( $\mathrm{pH} 11.0)$ (1/20 dilution), aliquoted and frozen at $-80^{\circ} \mathrm{C}$. Sandwich ELISA analysis of $A \beta_{42}$ in the obtained fractions was performed using Human $\mathrm{A} \beta_{42}$ ELISA Kit (Invitrogen) according to the manufacturer's instructions. Data were expressed in $\mathrm{pmol} / \mathrm{g}$ wet tissue.

\section{TTR Levels in Plasma and Supernatants of Cell Culture}

Mouse TTR (moTTR) in plasma of control and resveratrol-treated mice, as well as in the supernatants of primary cultures of hepatocytes, was quantified using Mouse Prealbumin ELISA Kit (MyBioSource) according to the manufacturer's instructions. Data were expressed in $\mathrm{mg} / \mathrm{L}$.

\section{QRT-PCR for TTR and LRPI Levels}

Total RNA from livers was isolated using Trizol (Alfagene ${ }^{\circledR}$ ). RNA concentrations were measured by NanoDrop 1000 spectrophotometer and stored at $-80^{\circ} \mathrm{C}$ until further use. For reverse transcription to cDNA, $4 \mu \mathrm{g}$ RNA was used with the SuperScript First-Strand Synthesis System (Invitrogen ${ }^{\mathrm{TM}}$ or Enzytech). The reaction mix was then subjected to quantitative real-time PCR with the SYBR Green reporter (iQ SYBR Green supermix, BioRad) to detect levels of moTTR and glyceraldehyde 3-phosphate dehydrogenase (GAPDH), as reference gene. The primers used were as follows: LRP-1 sense 5'-CGAGGA GCAGGTTGTTAG-3'; LRP-1 antisense 5'-CAGAAGCAGCAGGAGAAG-3'; moTTR sense: 5'-ATGAGAAGTTT GTAGAAGGAGTG-3'; moTTR antisense: 5'-AGAGTCGTTGGCTGTGAA-3'; GAPDH sense 5'-GCCTTCCGTGTTCCT ACC-3'; GAPDH antisense 5'-AGAGT GGGAGTTGCTGTTG-3'. Reactions were run in a Bio-Rad iCycler. The relative levels of expression were quantified by Bio-Rad iQ5 software. Data were calculated using the $\triangle \mathrm{CT}$ method, using GAPDH as a reference gene, before statistical analysis was performed.

\section{Assessment of TTR Tetrameric Stability}

Recombinant WT TTR $(333 \mu \mathrm{M})$ was incubated alone or in the presence of different compounds (iododiflunisal, diflunisal or resveratrol) for $1 \mathrm{~h}$ at $37^{\circ} \mathrm{C}$. Then, urea $8 \mathrm{M}$ and sample buffer without sodium dodecyl sulfate (SDS) were added. Samples were then run in a 15\% acrylamide gel prepared without SDS, and transferred onto a nitrocellulose membrane (Amersham ${ }^{\mathrm{TM}}$ GE Healthcare,
Protan $0.2 \mu \mathrm{m})$ using a wet system (Bio-Rad Criterion Blotter). The membranes were blocked for $1 \mathrm{~h}$ at RT with $5 \%$ nonfat dry milk (DM) in PBS containing $0.05 \%$ Tween-20 (PBS-T) and then incubated with primary antibody antihuman TTR (Dako) in 3\% DM/PBS-T. Then washed membranes were incubated for $1 \mathrm{~h}$ at RT with sheep antirabbit immunoglobulins conjugated with horseradish peroxidase (Binding Site; 1:5000 in 3\% DM/PBS-T). The blots were developed using Clarity ${ }^{\mathrm{TM}}$ Western ECL substrate (Bio-Rad), and levels of monomeric TTR were detected and visualized using a chemiluminescence detection system (ChemiDoc, Bio-Rad).

Alternatively, TTR alone or mixed with resveratrol, iododiflunisal or diflunisal at a molar ratio 1:10 (TTR:drug) was incubated at $37^{\circ} \mathrm{C}$ for $1 \mathrm{~h}$. Then, urea was added at $6 \mathrm{M}$ and samples were further incubated at $37^{\circ} \mathrm{C}$ overnight. The crosslinking reaction was performed by adding $2.5 \%$ glutaraldehyde for $4 \mathrm{~min}$, then the reaction was quenched by adding $0.1 \%$ sodium borohydride. Samples were then run on a $13.5 \%$ SDS-PAGE and transferred onto a nitrocellulose membrane, and treated as described above to detect folded TTR (tetramer + trimer + dimer).

\section{Thyroxine Binding Assays}

Qualitative studies of the displacement of $\mathrm{T}_{4}$ from WT TTR were carried out by incubating $5 \mu \mathrm{L}$ human plasma with ${ }^{125} \mathrm{I}_{-} \mathrm{T}_{4}$ (specific radioactivity $\approx 1200 \mu \mathrm{Ci} / \mu \mathrm{g}$; Perkin Elmer) in the presence of the different compounds (final concentration of $666 \mu \mathrm{M})$. Alternatively, $5 \mu \mathrm{l}$ plasma from nontreated mice or mice treated with resveratrol was used and handled as above. Protein separation was carried in a native PAGE system using glycine/acetate buffer. The gel was dried and revealed using an X-ray film.

\section{Western Blot for LRP 1}

The presence of LRP1 in brains was studied by western blot analysis. Protein extract of denatured samples 
(30-50 $\mu \mathrm{g})$ was separated in 15\% SDSPAGE gels and then transferred to nitrocellulose membrane (Amersham ${ }^{\mathrm{TM}}$ GE Healthcare, Protan $0.2 \mu \mathrm{m}$ ) using a wet system (Bio-Rad Criterion Blotter). The membranes were blocked for $1 \mathrm{~h}$ at RT with 5\% DM/PBS-T. After blocking, membranes were then incubated with primary antibodies in 3\% DM/PBS-T against the proteins under study: rabbit anti-LRP1 (Abcam, 1:10,000) and mouse anti-14-3-3 (pan) (Cell Signaling, $1: 10,000)$. Then washed membranes were incubated for $1 \mathrm{~h}$ at RT with sheep antirabbit (Binding Site; 1:5,000) or antimouse (Binding Site; 1:5000) immunoglobulins conjugated with horseradish peroxidase in 3\% DM/ PBS-T. The blots were developed using Clarity $^{\mathrm{TM}}$ Western ECL substrate (BioRad), and proteins were detected and visualized using a chemiluminescence detection system (ChemiDoc, Bio-Rad).

\section{Statistical Analyses}

D'Agostino and Pearson tests were used to evaluate normal distributions. The differences in brain $A \beta$ levels and plaque burden were analyzed by Mann Whitney test. A $p<0.05$ was considered significant for all analyses. GraphPad Prism version 5.04 for Windows (GraphPad Software) was the statistical software used.

\section{RESULTS}

\section{Resveratrol Administration Reduces A $\beta$ Brain Burden}

The effect of resveratrol in AD features was first assessed in terms of $A \beta$ deposition in the AD/TTR+/- mice by immunohistochemical analysis of brain sections using the 6E10 antibody, followed by quantification using Image Pro-Plus software. Results are depicted in Figure 1 and clearly show that resveratrol administration resulted in a significant diminishment of plaque burden in treated mice compared with control animals.

\section{Resveratrol Administration Reduces Total $A \beta_{42}$ Brain Levels}

We also evaluated the effect of resveratrol in the AD/TTR+/- mice by measuring $\mathrm{A} \beta_{42}$ levels in brain extracts in 2 different fractions, a detergent-soluble and an FA-soluble fraction, corresponding to the intra- and extracellular soluble $\mathrm{A} \beta$ (initial aggregates/oligomers), and to insoluble extracellular A $\beta$ (higher ordered aggregates of $A \beta$ ) (37), respectively. ELISA analysis showed that treatment with resveratrol resulted in a significant decrease in $A \beta$ levels in the detergent-soluble fraction (Figure 2A). As for the FA-soluble fraction, initial analysis including the 4 resveratrol-treated mice revealed that the differences in $A \beta_{42}$ between treated and nontreated mice were not statistically significant (Figure 2B). Of note, in the resveratrol-treated mice, the age at the beginning of the treatment was different within this group, and consequently the age at euthanization (see Table 1). At the time of death, treated animals were 10 months $(n=1), 8$ months $(n=2)$ or 7 months $(n=1)$ of age. Performing an age-dependent analysis on plaque burden and $A \beta$ brain levels (Figure 3), the 10-month-old mouse demonstrated increased levels of $A \beta_{42}$ in the FA-soluble $\mathrm{A} \beta$ fraction, presenting 2- and 8-fold increases in $A \beta$ levels, compared with the averages of the control group and the 7-month-old treated animals, respectively. Thus, considering only the younger treated mice, the differences between controls and treated animals were found to be statistically significant (Figure 2C). Nonetheless, still considering the 10-month-old mouse, resveratrol treatment induced a diminished plaque burden and lowered the levels of the $A \beta$ detergent-soluble fraction compared to the average of the control mice 7 months of age at the end of the experiment (Figure 3).

\section{Resveratrol Administration Increases TTR Protein Levels}

We then set out to assess whether the treatment using our AD mouse model

\section{A}

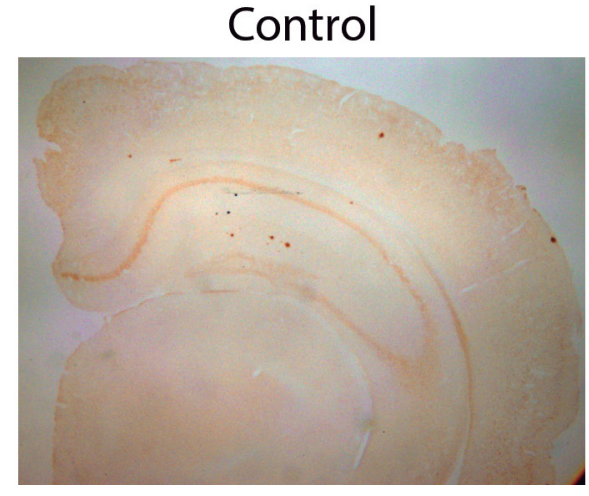

\section{Resveratrol-treated}

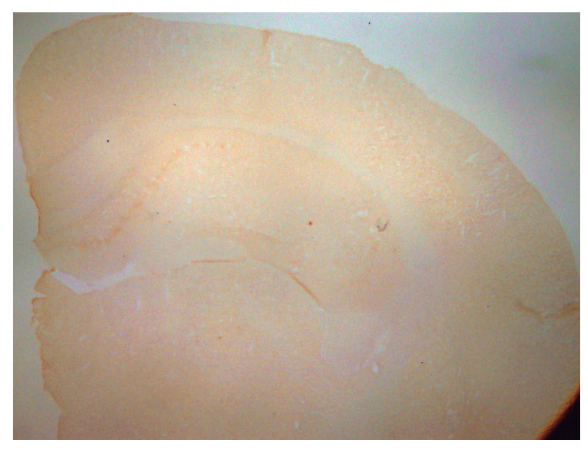

B

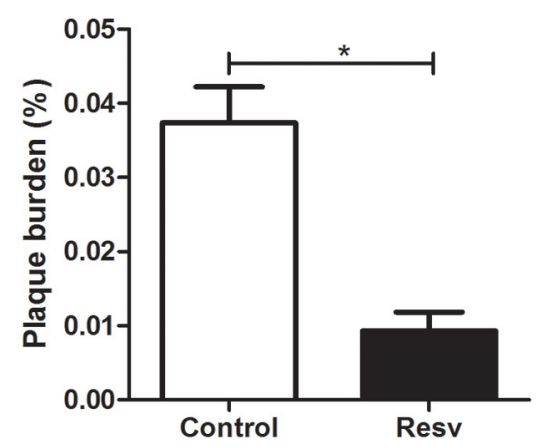

Figure 1. A $\beta$ plaque burden in $A D / T T R+/-$ mice, control or treated with resveratrol $(A)$ Photomicrographs illustrate immunohistochemical analysis of brain $A \beta$ plaques using the $6 E 10$ antibody. (B) $A \beta$ plaque burden in $A D / T T R+/-$ mice, control $(n=7)$ or treated with resveratrol (Resv) $(n=4)$. Error bars represent SEM. * $p<0.05$. 
A

\section{Detergent-soluble $A \beta 42$}

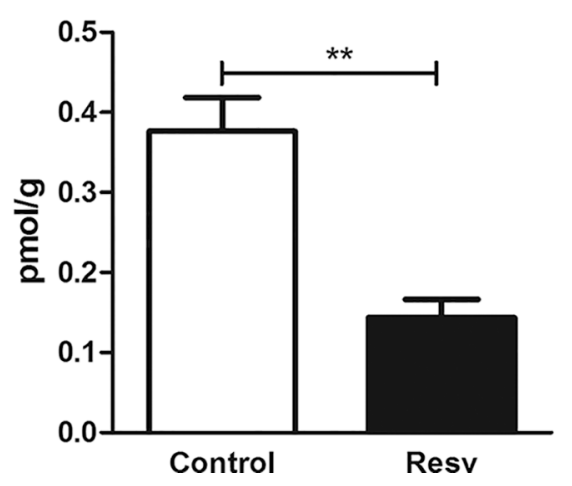

B

FA-soluble $A \beta 42$

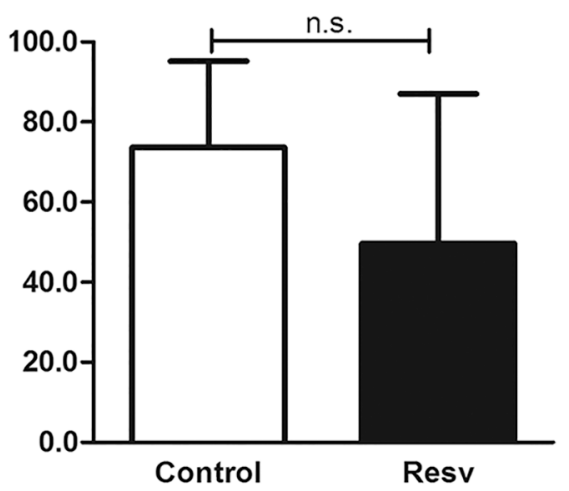

C

FA-soluble $A \beta 42$

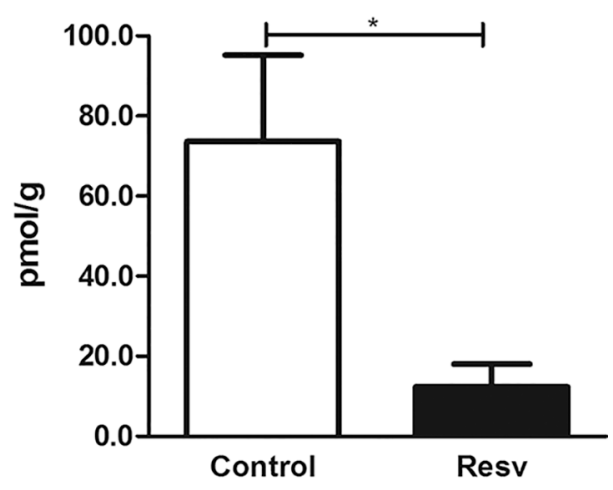

Figure 2. Brain $A \beta$ levels in $A D / T T R+/-$ mice, control or treated with resveratrol, quantified by ELISA. Levels of detergent-soluble $A \beta_{42}$ ( $A$ ) and FA-soluble $A \beta_{42}$ (B and $C$ ) were determined in brain extracts of control $(n=7)$ and treated mice (Resv) $(n=4$ in $A$ and $B, n=3$ in $C$ ). Error bars represent SEM. ${ }^{*} p<0.05 ;{ }^{* *} p<0.01$.

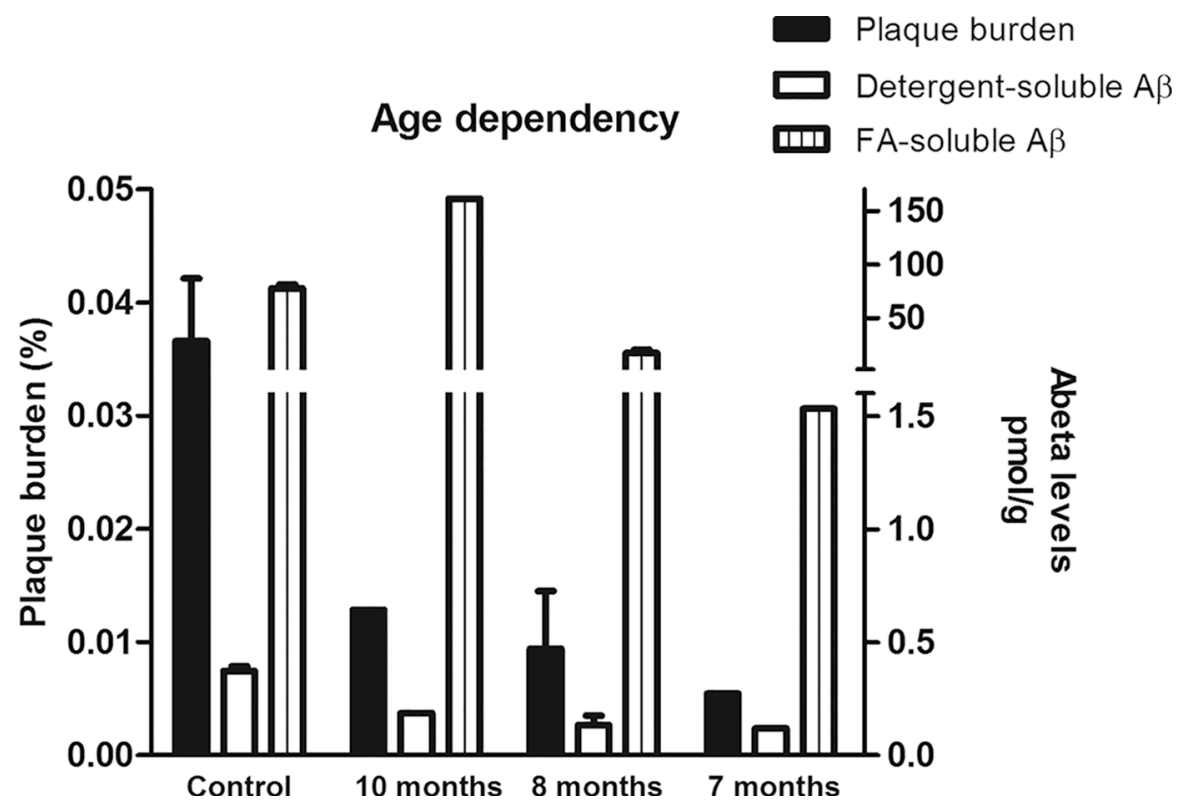

Figure 3. Age-dependent analysis of $A \beta$ deposition and $A \beta$ levels in $A D / T T R+/-$ mice, control or treated with resveratrol. Analyzing the results in an age-dependent manner, we observed that $A \beta$ plaque burden (left axis) and brain detergent-soluble $A \beta_{42}$ levels (right axis) had a tendency to decrease along with lower age. As for the FA-soluble fraction, we observed that for the older mouse, the treatment did not confer any protection, showing even higher levels than the average of the control group.

had any effect on TTR protein levels and gene expression, by measuring TTR plasma levels and TTR mRNA in the producer organ, the liver. TTR plasma levels were measured by ELISA and were significantly increased after
2 months of treatment with resveratrol (Figure 4A). Variation of TTR plasma levels with age is still controversial, and while some authors have shown increased levels (11), others have not observed any significant differences (38).
In our work, we did not find any statistically significant difference between mice of different ages (data not shown). Interestingly, TTR mRNA levels in the liver were not altered by resveratrol administration (Figure 4B), indicating that the effect was at the level of the protein.

The levels of TTR were also investigated by ELISA upon incubation of resveratrol with primary cultures of hepatocytes derived from TTR+/- mice (Figure 4C). Corroborating the results obtained from the in vivo treatment, after $24 \mathrm{~h}$ of incubation with resveratrol, the levels of TTR in the supernatant were increased compared with the control situation (absence of resveratrol). This observation supports our suggestion that the increased levels of TTR are not age-related but are due to the administration of resveratrol.

\section{Resveratrol Is Capable of Stabilizing TTR}

After observing that resveratrol had an effect on TTR protein levels, significantly increasing the levels of protein while not affecting the levels of its transcript, we set out to investigate whether this effect was due to increased stability of the protein. For that, we started with an in vitro approach, where we incubated human recombinant TTR with resveratrol and then subjected the mix to denaturation 
A

\section{TTR plasma levels}

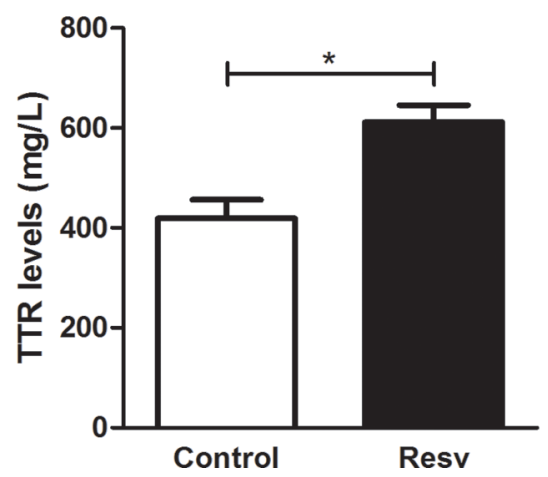

B

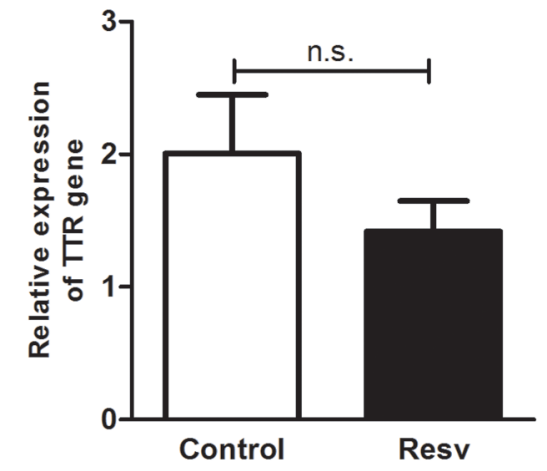

C

HepatocytesTTR levels

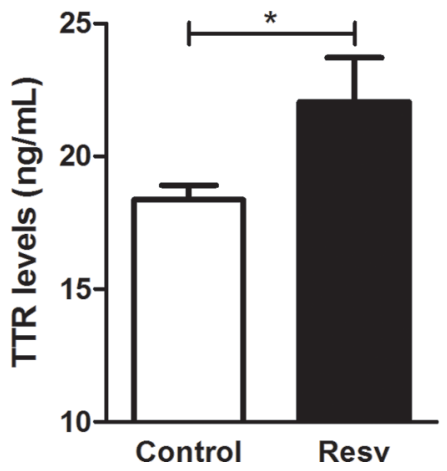

Figure 4. TTR protein and gene expression levels in AD/TTR+/- mice, control or treated with resveratrol (A) Significant differences were observed between control $(n=7)$ and treated mice (Resv) $(n=4)$ by ELISA, showing increased plasma TTR protein levels after resveratrol administration. (B) No significant alteration in gene expression was observed in livers of treated mice (Resv) ( $n=3)$, compared with control $(n=6)$. (C) TRR protein levels measured by ELISA were found to be increased in the supernatants of hepatocytes after resveratrol $(15 \mu \mathrm{M})$ incubation. ${ }^{*} p<0.05$.

A1

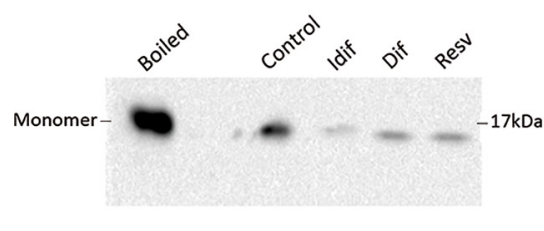

B

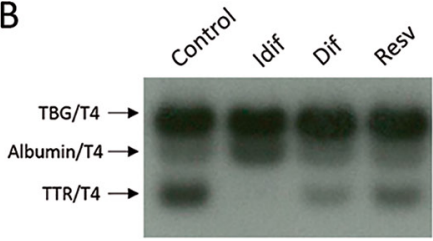

A2

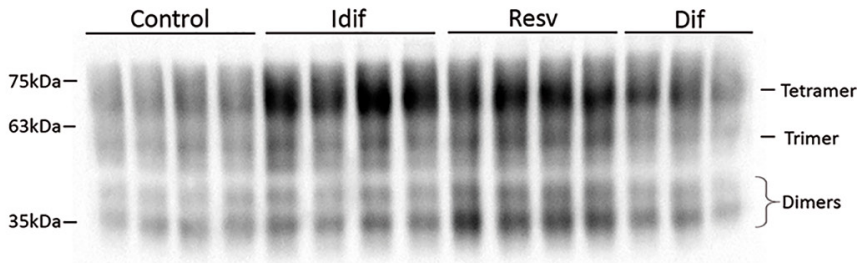

C

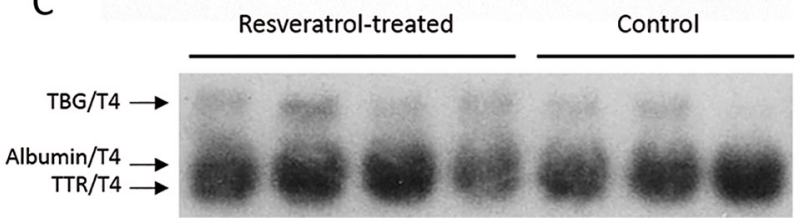

Figure 5. TTR stabilization by resveratrol (A) Western blot of human recombinant TTR showed increased TTR stability upon incubation with iododiflunisal, resveratrol and diflunisal. After urea denaturation, the analysis was performed either under semidenaturing conditions, revealing decreased monomeric TTR (A 1), or after cross-linking of the generated species, showing increased folded TTR (tetramer, trimer and dimer) (A2), compared with the control. (B) Native gel electrophoresis of human plasma, incubated with ${ }^{125} \mathrm{I}-\mathrm{T}_{4}$ and $\mathrm{competitor}$ showing that iododiflunisal, diflunisal and resveratrol compete with $\mathrm{T}_{4}$ for its binding site. (C) Native gel electrophoresis of control and resveratrol-treated mice plasma, incubated with ${ }^{125} \mathrm{I}-\mathrm{T}_{4}$, showing that resveratrol at its plasma concentration was not bound to TTR at the $\mathrm{T}_{4}$ binding site. TBG: thyroxine-binding globulin.

by urea, followed by a semidenaturating PAGE (Figure 5A1), to evaluate the levels of monomeric TTR. Alternatively, the mix underwent denaturation by urea and the generated species were crosslinked with glutaraldehyde, after which the samples were analyzed by a standard SDS-PAGE (Figure 5A2), to evaluate the levels of folded TTR (tetramer, trimer and monomer). In both cases, the final evaluation was done by western blot. To strengthen the result, we used iododiflunisal and diflunisal, both described as TTR stabilizers. As expected, incubation with iododiflunisal and diflunisal resulted in increased TTR stability, as deduced by a decrease in the intensity of the band corresponding to the monomer and an increase in the intensity of the bands corresponding to folded TTR. Interestingly, resveratrol also promoted an increase of folded TTR, although its effect is likely to be inferior to the one produced by iododiflunisal and more similar to the one created by diflunisal, as inferred by the amount of monomeric TTR. 
A

B

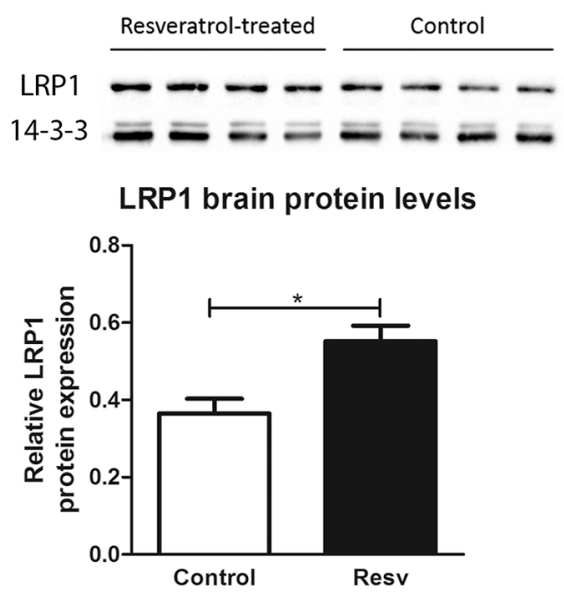

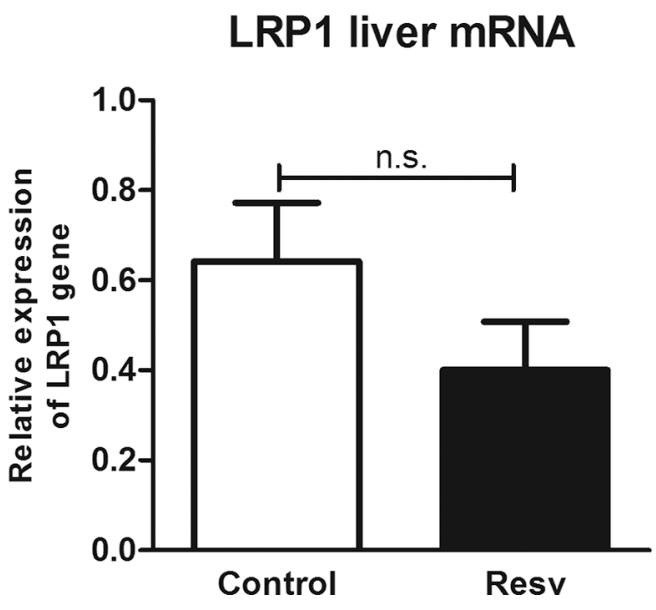

Figure 6. LRPI protein and gene expression levels in AD/TTR+/- mice, control or treated with resveratrol (A) Brain LRP1 protein levels were measured by western blot, and found to be increased upon treatment with resveratrol $(n=4)$, compared with control $(n=4)$. (B) Liver LRPI gene expression was measured by qRT-PCR and showed no difference between treated $(n=4)$ and control mice $(n=4)$. ${ }^{*} p<0.05$.

In addition, since it has been described that compounds that bind in the $\mathrm{T}_{4}$ binding pocket are prone to stabilize TTR, we tested the ability of resveratrol to compete with radiolabeled $\mathrm{T}_{4}$. In this assay we used human plasma as the source of TTR and measured the degree of competition with ${ }^{125} \mathrm{I}_{-} \mathrm{T}_{4}$ by evaluating the decrease in the intensity of the TTR $/{ }^{125} \mathrm{I}_{-} \mathrm{T}_{4}$ band. All the compounds were able to displace $T_{4}$, showing that resveratrol, although to a lesser extent than in our positive controls, was able to bind in the $\mathrm{T}_{4}$ binding pocket and displace $\mathrm{T}_{4}$ (Figure 5B). We then investigated if resveratrol competed with $\mathrm{T}_{4}$ in vivo, using plasma samples from control and resveratrol-treated mice. Again, a radiolabeled $\mathrm{T}_{4}$ assay was performed, showing no differences in the TTR $/{ }^{125} \mathrm{I}_{-} \mathrm{T}_{4}$ band between treated and nontreated animals, suggesting that, at least at the concentrations that resveratrol reached the plasma, the compound was not able to compete with $\mathrm{T}_{4}$ (Figure 5C).

\section{Resveratrol Administration Increases LRP 1 Protein Levels}

In a previous work, we showed that TTR modulates LRP1 levels in both the brain and liver, and we suggested that TTR-assisted A $\beta$ clearance is via LRP1. Thus, in this work we investigated LRP1 levels after resveratrol administration in brain homogenates by western blot (Figure 6A), which showed increased levels upon treatment with resveratrol. To further characterize the mechanism underlying resveratrol's effect in AD features, we assessed whether the gene expression was also being upregulated by the administration of resveratrol. We measured LRP1 mRNA levels in liver samples (Figure 6B) and observed that the treatment had no significant effect on expression of the LRP1 gene.

\section{DISCUSSION}

TTR is described as a transporter of $\mathrm{T}_{4}$ and retinol; however, more recently it has been shown to be a binding partner for $A \beta$ and suggested to be intimately involved in its clearance from the brain (17). As for resveratrol, apart from its potent antioxidant properties, several studies have demonstrated that this polyphenol also plays a neuroprotective role, although the mechanism by which this is performed is still unknown. Resveratrol bioavailability is low (less than
$1 \%$ when administered orally) (39), and therefore studies have been performed using high doses (50-300 mg/ kg/day) (40) in mice. The corresponding human dose, using a scaling factor of 0.08 , is approximately $1 \mathrm{~g}$. This concentration was found to be within the range of doses used in human studies, in which $1 \mathrm{~g}$ of resveratrol was shown to be well tolerated (41).

In this study, we conducted various experiments to study resveratrol's neuroprotective role, suggesting that the effects observed are, at least partially, TTR-dependent. It has been previously determined that amyloidogenic and unstable TTR mutants bind poorly to $A \beta$, suggesting that this interaction is dependent on TTR stability. Here, we show that in vivo administration of resveratrol $(0.19 \% \mathrm{w} / \mathrm{w})$ to 5 - to 8 -month-old AD/ TTR+/- mice, in which disease onset and $\mathrm{A} \beta$ deposition has been described to start around the age of 6 months, ameliorated the analyzed AD features. Thus, after 2 months of treatment, mice presented significantly lower levels of amyloid deposits, accompanied by a decrease in brain A $\beta$ peptide levels, both detergent- and FA-soluble fractions, compared to nontreated mice. Interestingly, regarding the older mouse (10 months old), the FA-soluble fraction of $A \beta$ was much higher than that observed in both the control and the rest of the treated group. These observations suggest that resveratrol performs best at inhibiting $\mathrm{A} \beta$ aggregation in the initial stages, rather than at removing already installed amyloid plaques. Previous work by Varamini and colleagues, using AD transgenic mice and resveratrol administrated at a dose similar to the one used in our study, failed to show decreased $A \beta$ deposition and $A \beta$ brain levels, as well as tau phosphorylation levels, although positive alterations in other markers were reported, such as glycogen synthase kinase 3- $\beta$ (GSK3- $\beta$ ) and developmentally regulated brain protein (drebrin) (30). This study was performed in older mice (11-13 months at the beginning of treatment), further supporting our observation in relation to 
the 10-month-old mouse, and reenforcing the need for early-stage treatments.

Resveratrol is known to bind TTR, avoiding its aggregation (42) through stabilization of the native tetramer. To understand the effect that the in vivo administration of resveratrol produced in TTR, we measured plasma TTR levels, which were increased in treated mice compared with the control group. This corroborated the results obtained by Varamini and colleagues (30), which described increased TTR protein levels measured in brain extracts. In addition to the in vivo studies, we established primary cultures from hepatocytes derived from TTR+/- mice, confirming the capacity of resveratrol to increase TTR protein levels. Since we found no differences in liver TTR mRNA, these results strengthen our hypothesis regarding the neuroprotective role of resveratrol via TTR stabilization, which appears to be most effective when administered before disease onset. Thus, the increase in TTR levels is due to its stabilization by resveratrol, consequently increasing the halflife time in circulation. A recent work with human TTR transgenic mice in the context of FAP showed that administration of a TTR stabilizer resulted in increased serum TTR levels and increased TTR tetrameric resistance to acid denaturation, without changing liver mRNA (43). In the AD context, higher levels of stabilized TTR would result in improved $\mathrm{A} \beta$ binding, therefore avoiding aggregation and deposition of the peptide. Thus, we could confirm the ability of resveratrol to improve TTR stability in vitro and investigate the underlying mechanism. Although resveratrol competed with $\mathrm{T}_{4}$ for TTR binding in vitro, using high resveratrol concentrations, plasma TTR from treated mice showed no $\mathrm{T}_{4}$ displacement compared with nontreated mice. This indicates either that resveratrol in plasma did not reach a concentration high enough to compete with $\mathrm{T}_{4}$ and, in this case, the beneficial effects in AD features were not necessarily TTRrelated, or that resveratrol binds TTR in another site. The 4 monomers within a TTR tetramer demarcate through the molecule an open channel where 2 binding sites for thyroid hormones are located $(44,45)$. These 2 binding sites present negative cooperativity (46), implying that when the first thyroid hormone molecule occupies the first site, affinity for the second molecule is greatly reduced. Interestingly, resveratrol, also characterized by negative binding cooperativity to TTR, binds preferentially to the other site, consistent with the data of X-ray analysis of TTR in complex with both ligands. (33) Although $\mathrm{T}_{4}$ displacement from TTR was detected at high concentrations, the concentration at which this polyphenol reached the plasma upon oral administration was likely not high enough to produce the same effect. Resveratrol is well known for its antioxidant proprieties and for acting as an antitumor (47), anti-aging (48) and antineurodegeneration (28) agent. Therefore, it is important to consider that the effects observed in AD features, namely in $\mathrm{A} \beta$ levels and aggregation state, may be a combination of effects.

We also analyzed the levels of LRP1, known to be reduced in AD patients. We assessed protein and gene expression levels of this receptor and found increased protein levels in the brains of the resveratrol-treated animals, while its gene expression values did not present any differences in the liver when comparing treated and nontreated animals. Our previous work has shown that TTR modulates LRP1 levels, in both the brain and the liver, impacting both in protein and mRNA levels, as analyzed in nontransgenic animals. (17) Our present results might indicate an indirect action of TTR in LRP1, via its stabilization by resveratrol. For instance, it has been described that $\mathrm{A} \beta$ peptide promotes LRP1 degradation at the proteasome (49). The decrease in $A \beta$ levels induced by TTR, which were increased due to increased stability and therefore reduced/ normalized clearance, resulted in increased LRP1.

Although administration of resveratrol was undoubtedly very effective in our animal model for AD while showing a TTR-dependent mechanism, its bioavailability is very low. Therefore, it would be very interesting to test polyphenol metabolites, since their bioavailability is much higher compared with nonmetabolized polyphenols; for example, resveratrol-3-O-sulfate presents similar stabilization and inhibition of fibrillization of TTR compared with resveratrol (33). In addition, other polyphenols present in wine in much larger quantities should be assessed.

\section{CONCLUSION}

Altogether, our results indicate that resveratrol stabilizes TTR, thus increasing its circulating levels. In turn, the stabilized TTR presents an improved ability to bind $\mathrm{A} \beta$ peptide, which avoids its aggregation and deposition, thus explaining the amelioration in AD features in vivo.

\section{DISCLOSURE}

The authors declare that they have no competing interests as defined by Molecular Medicine or other interests that might be perceived to influence the results and discussion reported in this paper.

\section{ACKNOWLEDGMENTS}

This work was financed by Fundo Europeu de Desenvolvimento Regional funds through the COMPETE 2020 Operacional Programme for Competitiveness and Internationalisation, Portugal 2020, and by Portuguese funds through Fundação para a Ciência e a Tecnologia/ Ministério da Ciência, Tecnologia e Inovação, in the framework of the project Institute for Research and Innovation in Health Sciences (POCI-01-0145FEDER-007274). The work was also funded by Fundacion La Marató de Tv3, Spain, through project 20140330-3132-33-34 and by the Portuguese Institute for the Vine and Wine. I.C. works under the Investigator FCT Program, which is financed by national funds through the Foundation for Science and Technology and co-financed by the European Social 
Fund through the Human Potential Operational Programme, type 4.2, Promotion of Scientific Employment. M.A. is the recipient of a research fellowship from IBMC, funded by project of Fundacion La Marató de Tv3, and L.M.S. was the recipient of a research fellowship from IBMC funded by the International Organization of Vine and Wine.

\section{REFERENCES}

1. Alzheimer A, Stelzmann RA, Schnitzlein HN Murtagh FR. (1995) English translation of Alzheimer's 1907 paper, Uber eine eigenartige Erkankung der Hirnrinde. Clin. Anat. 8:429-31

2. Brookmeyer R, Johnson E, Ziegler-Graham K Arrighi HM. (2007) Forecasting the global burden of Alzheimer's disease. Alzheimer's Dement. 3:186-91.

3. Alzheimer's disease facts and figures. (2015) Alzheimer's Dement. 11:332-84.

4. Pimplikar SW. (2009) Reassessing the amyloid cascade hypothesis of Alzheimer's disease. Int. J. Biochem. Cell Biol. 41:1261-68.

5. Shibata M, et al. (2000) Clearance of Alzheimer's amyloid-ss (1-40) peptide from brain by LDL receptor-related protein-1 at the blood-brain barrier. J. Clin. Invest. 106:1489-99.

6. Tarasoff-Conway JM, et al. (2015) Clearance systems in the brain: implications for Alzheimer disease. Nat. Rev. Neurol. 11:457-70.

7. Pascale CL, et al. (2011) Amyloid-beta transporter expression at the blood-CSF barrier is age-dependent. Fluids Barriers CNS. 8:21.

8. Schwarzman AL, et al. (1994) Transthyretin sequesters amyloid beta protein and prevents amyloid formation. Proc. Natl. Acad. Sci. U.S.A. 91:8368-72.

9. Schwarzman AL, Goldgaber D. (1996) Interaction of transthyretin with amyloid beta-protein: binding and inhibition of amyloid formation. Ciba Found. Symp. 199:146-60; discussion 160-64.

10. Ribeiro CA, et al. (2012) Transthyretin decrease in plasma of $\mathrm{MCI}$ and $\mathrm{AD}$ patients: investigation of mechanisms for disease modulation. Curr. Alzheimer Res. 9:881-89.

11. Serot JM, Christmann D, Dubost T, Couturier M. (1997) Cerebrospinal fluid transthyretin: aging and late onset Alzheimer's disease. J. Neurol. Neurosurg. Psychiatry 63:506-8.

12. Merched A, et al. (1998) Apolipoprotein E, transthyretin and actin in the CSF of Alzheimer's patients: relation with the senile plaques and cytoskeleton biochemistry. FEBS Lett. 425:225-28.

13. Gloeckner SF, et al. (2008) Quantitative analysis of transthyretin, tau and amyloid-beta in patients with dementia. J. Alzheimer's Dis. 14:17-25.

14. Heywood WE, et al. (2015) Identification of novel CSF biomarkers for neurodegeneration and their validation by a high-throughput multiplexed targeted proteomic assay. Mol. Neurodegener. 10:64.
15. Choi SH, et al. (2007) Accelerated Abeta deposition in APPswe/PS1deltaE9 mice with hemizygous deletions of TTR (transthyretin). J. Neurosci. 27P7006-10.

16. Oliveira SM, Ribeiro CA, Cardoso I, Saraiva MJ. (2011) Gender-dependent transthyretin modulation of brain amyloid- $\beta$ levels: evidence from a mouse model of Alzheimer's disease. J. Alzheimer's Dis. 27, 429-39.

17. Alemi M, et al. (2016) Transthyretin participates in beta-amyloid transport from the brain to the liver: involvement of the low-density lipoprotein receptor-related protein 1? Sci. Rep. 6:20164.

18. Costa R, Gonçalves A, Saraiva MJ, Cardoso I. (2008) Transthyretin binding to A-Beta peptide: impact on A-Beta fibrillogenesis and toxicity. FEBS Lett. 582,:936-42.

19. Ribeiro CA, et al. (2014) Transthyretin stabilization by iododiflunisal promotes amyloid- $\beta$ peptide clearance, decreases its deposition, and ameliorates cognitive deficits in an Alzheimer's disease mouse model. J. Alzheimer's Dis. 39:357-70.

20. Du J, Murphy RM. (2010) Characterization of the interaction of $\beta$-amyloid with transthyretin monomers and tetramers. Biochemistry. 49: 8276-89.

21. Li X, et al. Mechanisms of transthyretin inhibition of $\beta$-amyloid aggregation in vitro. J. Neurosci. 33:19423-33.

22. Cascella R, et al. Transthyretin suppresses the toxicity of oligomers formed by misfolded proteins in vitro. Biochim. Biophys. Acta - Mol. Basis Dis. 1832:2302-14.

23. Mannini B, et al. (2012) Molecular mechanisms used by chaperones to reduce the toxicity of aberrant protein oligomers. Proc. Natl. Acad. Sci. 109:12479-84

24. Renaud S, de Lorgeril M. (1992) Wine, alcohol, platelets, and the French paradox for coronary heart disease. Lancet. 339:1523-26.

25. Rocha-González H, Ambriz-Tututi M, Granados-Soto V. (2008) Resveratrol: a natural compound with pharmacological potential in neurodegenerative diseases. CNS Neuroscience and Therapeutics. 14:234-47.

26. Li F, Gong Q, Dong H, Shi J. (2012) Resveratrol, A Neuroprotective Supplement for Alzheimer's Disease. Curr. Pharm. Des. 18:27-33.

27. Kim D, et al. (2007) SIRT1 deacetylase protects against neurodegeneration in models for Alzheimer's disease and amyotrophic lateral sclerosis. EMBO J. 26:3169-79.

28. Karuppagounder SS, et al. (2009) Dietary supplementation with resveratrol reduces plaque pathology in a transgenic model of Alzheimer's disease. Neurochem. Int. 54:111-18.

29. Marambaud P, Zhao H, Davies P. (2005) Resveratrol promotes clearance of Alzheimer's disease amyloid-beta peptides. J. Biol. Chem. 280:37377-82.

30. Varamini B, Sikalidis AK, Bradford KL. (2013) Resveratrol increases cerebral glycogen synthase kinase phosphorylation as well as protein levels of drebrin and transthyretin in mice: an exploratory study. Int. J. Food Sci. Nutr. 7486:1-8.

31. Ribeiro CA, Saraiva MJ, Cardoso I. (2012) Stability of the Transthyretin Molecule as a Key Factor in the Interaction with A-Beta Peptide: Relevance in Alzheimer's Disease. PLoS One. 7_e45368.

32. Klabunde $\mathrm{T}$, et al. (2000) Rational design of potent human transthyretin amyloid disease inhibitors. Nat. Struct. Biol. 7:312-21.

33. Florio $\mathrm{P}$, et al. Transthyretin binding heterogeneity and antiamyloidogenic activity of natural polyphenols and their metabolites. J. Biol. Chem. 290:29769-80.

34. Furuya H, et al. (1991) Production of recombinant human transthyretin with biological activities toward the understanding of the molecular basis of familial amyloidotic polyneuropathy (FAP). Biochemistry. 30:2415-21.

35. Almeida MR, Damas AM, Lans MC, Brouwer A Saraiva MJ. (1997) Thyroxine binding to transthyretin Met 119. Comparative studies of different heterozygotic carriers and structural analysis. Endocrine. 6:309-15.

36. Episkopou V, et al. (1993) Disruption of the transthyretin gene results in mice with depressed levels of plasma retinol and thyroid hormone. Proc. Natl. Acad. Sci. U.S.A. 90:2375-79.

37. Steinerman JR, et al. (2008) Distinct pools of beta-amyloid in Alzheimer disease-affected brain: a clinicopathologic study. Arch. Neurol. 65:906-12.

38. Kunicki S, Richardson J, Mehta PD, Kim KS, Zorychta E. (1998) The effects of age, apolipoprotein $\mathrm{E}$ phenotype and gender on the concentration of amyloid-beta (A beta) 40, A beta 4242, apolipoprotein $\mathrm{E}$ and transthyretin in human cerebrospinal fluid. Clin. Biochem. 31:409-15.

39. Walle T. (2011) Bioavailability of resveratrol. Ann. N.Y. Acad. Sci. 1215:9-15.

40. Robb EL, Stuart JA. (2010) Trans-resveratrol as a neuroprotectant. Molecules. 15:1196-212.

41. Patel KR, et al. (2010) Clinical pharmacology of resveratrol and its metabolites in colorectal cancer patients. Cancer Res. 70:7392-99.

42. Bourgault S, et al. (2011) Mechanisms of transthyretin cardiomyocyte toxicity inhibition by resveratrol analogs. Biochem. Biophys. Res. Commun. 410:707-13.

43. Mu Y, et al. (2015) CHF5074 (CSP-1103) stabilizes human transthyretin in mice humanized at the transthyretin and retinol-binding protein loci. FEBS Lett. 589:849-56.

44. Blake CCF, Geisow MJ, Swan IDA, Rerat C, Rerat B. (1974) Structure of human plasma prealbumin at 2.5 A resolution. A preliminary report on the polypeptide chain conformation, quaternary structure and thyroxine binding. J. Mol. Biol. 88

45. Blake CC, Geisow MJ, Oatley SJ, Rérat B, Rérat C. Structure of prealbumin: secondary, tertiary and quaternary interactions determined by Fourier refinement at 1.8 A. J. Mol. Biol. 121:339-56. 
46. Ferguson RN, Edelhoch H, Saroff HA,

Robbins J, Cahnmann HJ. (1975) Negative cooperativity in the binding of thyroxine to human serum prealbumin. Preparation of tritium-labeled 8-anilino-1-naphthalenesulfonic acid. Biochemistry. 14:282-89.

47. Kimura Y, Okuda H. (2001) Resveratrol isolated from Polygonum cuspidatum root prevents tumor growth and metastasis to lung and tumor induced neovascularization in Lewis lung carcinoma bearing mice. J. Nutr. 131:1844-49.

48. Gruber J, Soon YT, Halliwell B. (2007) Evidence for a trade-off between survival and fitness caused by resveratrol treatment of Caenorhabditis elegans. In: Ann. N.Y. Acad. Sci. 1100:530-42.

49. Bates KA, et al. (2009) Clearance mechanisms of Alzheimer's amyloid-beta peptide: implications for therapeutic design and diagnostic tests. Mol. Psychiatry. 14:469-86.

Cite this article as: Santos LM, et al. (2016) Resveratrol administration increases transthyretin protein levels, ameliorating $\mathrm{AD}$ features: The importance of transthyretin tetrameric stability. Mol. Med. 22:597-607. 